\title{
Factores asociados al consumo de drogas en una muestra de militares españoles desplegados en "Bosnia-Herzegovina"
}

\section{Factors associated with substance use among Spanish military personnel involved in "Bosnia-Herzegovina"}

\author{
Cristina Vargas*, Enrique Castellano**, Humberto Trujillo**. \\ *Universidad de Valencia; **Universidad de Granada.
}

\section{Resumen}

El consumo de drogas legales e ilegales ha sido muy poco investigado en población militar española destinada en zonas de operaciones multinacionales. El objetivo de la investigación fue evaluar el nivel de consumo de drogas en militares españoles que realizaban misiones en Bosnia-Herzegovina, e identificar las posibles variables asociadas con dicho consumo. En el estudio transversal participaron 605 militares. La edad media de los participantes fue de 25,9 años $(D T=5,9)$ y el 93,9\% de la muestra eran hombres. La mayoría de los participantes pertenecían a las escalas de tropa y marinería $(83,5 \%)$. Las drogas más usadas fueron el tabaco (54,2\%), y el alcohol (39,9\%). En relación a las drogas ilegales, los resultados muestran que la droga con una mayor prevalencia de consumo "alguna vez en su vida" fue el cannabis $(36,2 \%)$, seguida de la cocaína $(14,9 \%)$ y las anfetaminas $(12,1 \%)$. La variable más relevante asociada con una disminución en el consumo de drogas ilegales ha sido el apoyo social. En cambio, los participantes quienes tenían amigos consumidores de drogas ilegales incrementaban la probabilidad de consumo de drogas. Se resalta la importancia de la prevención en zonas de operaciones multinacionales para evitar el efecto negativo que podría tener el consumo de drogas en el desempeño adecuado de las misiones encomendadas.

Palabras clave: Personal militar; Zonas de operaciones multinacionales; Consumo de drogas; Factores de riesgo; Factores de protección.

\begin{abstract}
The use of both legal and illegal drugs has rarely been investigated among the Spanish military population involved in multinational military operations. The aim of the current study was to examine the consumption of drugs by Spanish military personnel in BosniaHerzegovina, and the variables associated with such substance use. A total of 605 military personnel participated in the cross-sectional study. The participants' mean age was 25.9 years $(S D=5.9)$, and $93.9 \%$ of the sample was male. The majority of the participants were enlisted personnel $(83.5 \%)$. The most widely used drugs were tobacco $(54.2 \%)$, and alcohol (39.9\%). With respect to illegal drugs, the results showed that the drug with the highest prevalence of use "at some point during a lifetime" was cannabis $(36.2 \%)$, followed by cocaine $(14.9 \%)$ and amphetamines (12.1\%). The most important variable associated with a decrease in the consumption of illegal drugs was social support. Conversely, participants with friends who have used illegal drugs had an increased likelihood of drug consumption. Given that the use of drugs can adversely affect soldiers' performance, preventive measures should be applied in multinational military operations.

Keywords: Military personnel; Multinational military operations; Drug use; Risk factors; Protective factors.
\end{abstract}


$\mathrm{E}$ 1 consumo de drogas es un problema social que afecta a diversos colectivos, habiendo sido algunos de ellos más estudiados que otros. Por ejemplo, las investigaciones o informes elaborados sobre el consumo de drogas entre civiles adultos o adolescentes son numerosas tanto en el contexto nacional como internacional (Melchior, Chastang, Goldberg y Fombonne, 2008; Miquel et al., 2015; Motos Sellés, Cortés Tomás, Giménez Costa y Cadaveira Mahía, 2015; Mounteney et al., 2016; Observatorio Español sobre Drogas, 2013; Observatorio Español de la Droga y las Toxicomanías, 2015; Observatorio Europeo de las Drogas y las Toxicomanías, 2014). En cambio, otros colectivos más específicos han sido menos estudiados, entre ellos, la población militar. Las investigaciones realizadas en las Fuerzas Armadas españolas, que sepamos, son escasas y los resultados encontrados no suelen acompañarse de valores numéricos precisos con el objetivo de proteger la confidencialidad de información crítica en este colectivo (por ejemplo, revisar trabajo realizado por Donoso Rodríguez, 2012). En territorio nacional, Martínez, Alonso, Taranco y Gutiérrez (2010) realizaron un estudio sobre consumo de drogas ilegales con miembros no desplegados de las Fuerzas Armadas de los Ejércitos de Tierra, de la Armada y Aire. La investigación se inició en el año 2002 y finalizó el 2007. En general, encontraron que un $9 \%$ de los militares consumían actualmente cannabis, un $2 \%$ alucinógeno, un $3,5 \%$ anfetamina y un $8 \%$ cocaína. La prevalencia del consumo de drogas se fue reduciendo a lo largo de los años, a excepción del año 2003 donde se produjo un incremento en el consumo respecto al año 2002. Esto es, un 8,2\% consumieron cocaína en el 2002 reduciéndose a un 4,5\% en el 2007, este descenso progresivo no se mantuvo en el 2003 donde hubo un consumo de un $10,7 \%$. En otro estudio con población militar no desplegada, Donoso Rodríguez (2012) evaluó la prevalencia del consumo actual de drogas legales (alcohol y tabaco) e ilegales (cannabis, cocaína, opiáceos, alucinógenos y anfetaminas) en una muestra de tropa profesional no desplegada del Ejército de Tierra desde 1997 hasta 2007, y en un colectivo de cuadros de mando no desplegados desde 2002 hasta 2008. Este autor encontró que las sustancias más consumidas en la tropa profesional eran el alcohol y el tabaco, y de las sustancias ilegales, el cannabis, seguido de la cocaína y las anfetaminas. Si bien, un alto porcentaje de la tropa declaró no haber probado ningún tipo de sustancia psicoactiva ilegal. A lo largo de los años estudiados, se produjo un descenso progresivo y sustancial en el consumo de drogas legales e ilegales (cannabis, cocaína y anfetaminas). En relación a los militares pertenecientes a los cuadros de mando, las sustancias más consumidas seguían siendo las drogas legales (el alcohol y tabaco), mientras que el consumo de las ilegales era muy minoritario. La evolución del consumo se mantuvo estable a lo largo de los años y fue similar al de la tropa.
Algunos estudios sugieren que el consumo de drogas podría diferir entre personal militar y población civil. Por ejemplo, Teachman, Anderson y Tedrow (2015) encontraron que se producía un incremento en el consumo de alcohol en militares hombres que se alistaban en el ejército frente aquellos que no se enrolaban. Resultados opuestos ocurren cuando se trata de mujeres, la probabilidad de consumo de alcohol disminuía en aquellas mujeres que se alistaban en el ejército. Estos datos son de gran interés pues parecen indicar que podría haber una interacción entre la variable estar alistado y sexo. Es importante resaltar que la población militar española se caracteriza por estar constituida principalmente por hombres. Concretamente, el $83,5 \%$ del personal militar profesional de tropa y marinería son hombres, disminuyendo el porcentaje al 73,7\% cuando se trata de personal militar de complemento (Unidad de Estadística del Órgano Central, 2016).

De acuerdo con esta posible discrepancia en el consumo de drogas entre población militar y civil, en la Encuesta Domiciliaria sobre Alcohol y Drogas en España (EDADES) obtienen que la población civil española (de 15 a 64 años) ha consumido en los últimos 30 días un $6,6 \%$ de cannabis, un $0,1 \%$ de alucinógenos, un $0,3 \%$ de anfetaminas y un $1,1 \%$ de cocaína (Observatorio Español de la Droga y las Toxicomanías, 2015). Estas prevalencias de consumo de drogas ilegales son inferiores a los obtenidos en el estudio de Martínez et al. (2010). Bien es cierto que en ambos trabajos no utilizan la misma medida de consumo, aunque si son muy próximas (consumo actual y consumo en los últimos 30 días).

$\mathrm{Al}$ mismo tiempo, existen situaciones específicas que forman parte de la vida militar, como la participación en misiones internacionales en zonas de operaciones multinacionales con o sin exposición al combate, y que pueden estar asociadas con un incremento o consumo intensivo de drogas tradicionales como es el alcohol (Cucciare et al., 2015; Jacobson et al., 2008). Así, Kelsall et al. (2015) realizaron un estudio meta-analítico donde se comparaba el consumo de alcohol u otras sustancias entre militares que han participado en la Guerra del Golfo o en la Guerra de Iraq-Afganistán frente a otros compañeros que no han participado en dichos conflictos. Los militares que han participado en los combates mencionados previamente tenían un mayor riesgo de consumo de alcohol (OR de 1,3 en la Guerra del Golfo; OR de 1,4 en la Guerra de Iraq-Afganistán) o de otras sustancias (OR de 1,1 en la Guerra de Iraq-Afganistán).

Ya que los estudios realizados con población militar perteneciente a las Fuerzas Armadas españolas son escasos, sobre todo los llevados a cabo en zonas de operaciones multinacionales, sería importante disponer de información precisa sobre la prevalencia del consumo de drogas en dicho colectivo durante una misión internacional. Igualmente, sería de gran interés evaluar los posibles factores 
asociados con dicho consumo en este contexto y que previamente han sido significativos en otros colectivos (p. ej., Brook, Saar, Zhang y Brook, 2009; Coomber et al., 2011; Rudzinski et al., 2014; Schnohr et al., 2004, Sordo et al., 2015).

En consecuencia, el objetivo de este trabajo es evaluar el consumo de drogas legales e ilegales en una población militar española destinada en la zona de operaciones multinacionales de Bosnia-Herzegovina así como identificar las posibles variables asociadas con dicho consumo.

\section{Método}

\section{Participantes y procedimiento}

La muestra estaba formada por 605 militares profesionales pertenecientes a las Fuerzas Armadas españolas (Ejército de Tierra e Infantería de Marina), destinados en unidades operativas en la zona de operaciones multinacionales ubicadas todas ellas en Bosnia-Herzegovina (bases de Mostar-España, Mostar-Aeropuerto, destacamento Duzi en Trebinje, y bases de Sarajevo y Raylovac). El total de militares que componían el contingente en el intervalo de tiempo en el que se llevó a cabo la investigación era de 1212. El criterio de inclusión fue que los participantes estuvieran libres de tareas propias de su actividad laboral en el momento de ser evaluados. Es decir, la muestra incidental estaba constituida por todos los militares que componían el contingente con la excepción de aquellos que estaban realizando, en el momento de la aplicación de los cuestionarios, trabajos específicos tales como escolta, guardias, vigilancia, etc. Por tanto, de la totalidad de los militares desplegados en dicho contingente, estuvieron disponibles el $49,9 \%$, y todos ellos, aceptaron participar de forma voluntaria.

El estudio realizado fue transversal. El cuadernillo de evaluación fue auto-administrado de forma colectiva todos los miércoles, desde enero hasta abril de 2003, a las 11.00 de la mañana en los comedores de los acuartelamientos. En la sala, siempre estuvo presente el mismo investigador que iniciaba la sesión explicando en voz alta las instrucciones para cumplimentar el cuadernillo y aclarando cualquier duda que surgiera antes de que los participantes empezaran a rellenarlo. La sesión duraba aproximadamente 60 minutos. Se advirtió de la importancia de no dejar ninguna pregunta o ítem sin responder. Por último, se garantizó el anonimato de los participantes y la confidencialidad de la información obtenida. Todos los militares presentes en el momento de la aplicación de la encuesta participaron voluntariamente en la investigación y no recibieron ninguna compensación por ello.

\section{Variables}

Se administró un cuadernillo con el que se obtuvo información relativa a las características sociodemográficas (edad, sexo, nivel de estudios, con quién viven, localidad de origen, etc.), hábitos de consumo de drogas legales en familiares (consumo de tabaco en padres y hermanos), amigos (consumo de drogas ilegales) y consumo de distintas drogas en los propios encuestados (alcohol, tabaco, cannabis, anfetaminas, cocaína y otras sustancias). Concretamente, se registró consumo habitual de alcohol (¿bebe alcohol habitualmente?), consumo actual de tabaco (¿fuma en la actualidad?) y si se ha consumido alguna vez en su vida drogas ilegales (por ejemplo, ¿en alguna ocasión ha probado cocaína?). Así mismo, se incluyeron preguntas específicas del ámbito militar como el tipo de unidad militar de la que procede (fuerza, apoyo a la fuerza y cuartel general/plana mayor) o escala militar (tropa y mandos).

Las variables de protección fueron evaluadas mediante una adaptación del Cuestionario de Factores de Riesgo y Protección (CFR-P; Martínez-González, Trujillo-Mendoza y Robles-Lozano, 2007) para su aplicación a la población objeto de estudio. Este instrumento de medida fue incluido en el cuadernillo mencionado previamente, y está constituido por 35 ítems que evalúan variables de protección ante el consumo de drogas legales e ilegales. El formato de respuesta es de tipo Likert de cinco puntos, donde 1 equivale a nada de protección y 5 , máxima protección. Las propiedades psicométricas del cuestionario fueron evaluadas pues la muestra objeto de estudio era diferente en composición y variabilidad a la de referencia (Wilkinson y APA Task Force on Statistical Inference, 1999). Para el estudio de la estructura dimensional del instrumento de evaluación se realizó un análisis factorial exploratorio. Los resultados indicaron la existencia de dos dimensiones de protección que fueron habilidad de afrontamiento (23 ítems) y apoyo social (12 ítems). La consistencia interna obtenida mediante el coeficiente alfa de Cronbach para la dimensión habilidad de afrontamiento fue de 0,86 y para el apoyo social de 0,68 .

\section{Análisis estadísticos}

Los datos fueron analizados descriptivamente aplicando los estadísticos media aritmética y desviación típica para variables consideradas continuas, calculándose las frecuencias para las variables categóricas.

El estadístico t-Student fue usado para realizar los contrastes de hipótesis sobre dos medias de muestras independientes cuando la variable era cuantitativa. Si el supuesto de varianzas poblacionales iguales (comprobación mediante la prueba de Levene) no se asumía, se utilizaba la aproximación de Welch para realizar el contraste sobre diferencias de medias. El análisis chi-cuadrado de Pearson se aplicó cuando la variable era cualitativa y se contrastaba la prevalencia de consumo obtenida en las sustancias psicoactivas consideradas. Todas las variables que mostraron diferencias estadísticamente significativas con un p-valor < 0,05 se incluyeron como predictoras en los distintos análi- 
sis de regresión logística binaria múltiple realizados para cada una de las drogas legales e ilegales. El Odds Ratio de prevalencia (OR) era el indicador en el que se centraba el análisis multivariante. Cuando la variable predictora introducida en el modelo es cuantitativa, por ejemplo la edad (años cumplidos), y se obtiene un OR superior a 1 con respecto a la variable criterio, por ejemplo consumo de alcohol, significa que la probabilidad de consumo de alcohol que tiene un individuo de 35 años es mayor que la que tiene uno de 34 años. En cambio, cuando la OR es inferior a 1, la probabilidad de consumo de alcohol que tiene un individuo de 35 años es menor que la que tiene uno de 34 años. En la selección de variables se empleó el método por etapas hacia atrás (el criterio de eliminación se basó en la probabilidad del estadístico de Wald). Todos los análisis se realizaron con el paquete estadístico SPSS 20.0 con la excepción del cálculo de los intervalos de confianza al 95\% de las prevalencias de consumo de drogas, para lo cual se siguieron las indicaciones de Newcombe (1998).

\section{Resultados}

\section{Características de la muestra}

La edad media de los participantes fue de 25,9 años (DT $=5,9)$, siendo el rango de 18 a 51 años. El 93,9 \% de la muestra eran hombres y el $69,8 \%$ habían cursado estudios secundarios. La unidad de la que procedían mayoritariamente los participantes era la de fuerza $(54,7 \%)$ y el $83,5 \%$ de los militares pertenecían a la escala de tropa (Tabla 1).

\section{Prevalencias del consumo de drogas legales e ilegales}

Del total de la muestra, el 39,9\% consume habitualmente alcohol y el 54,2\% usan tabaco actualmente. En relación con las drogas ilegales, el 36,2\% informa haber probado alguna vez en su vida cannabis, el 12,1\% anfetaminas, y el 14,9\% cocaína. Sólo el $8 \%$ de los participantes informa haber probado alguna vez en su vida otras sustancias distintas a las indicadas (Tabla 2).

Cabe hacer explícito que los padres de los participantes consumen tabaco en un $41,7 \%$, las madres en un $21,9 \%$, y los hermanos en un 54,8\%. El consumo de drogas ilegales por parte de los amigos es de un 60,8\% (Tabla 1).

\section{Asociación entre variables de interés y consumo de drogas}

Las variables que resultaron estadísticamente significativas en relación al consumo habitual de alcohol fueron: el nivel de estudios, con quién viven, escala militar, padre consume tabaco, amigos consumen drogas ilegales y edad. Las variables que predicen el consumo habitual de alcohol fueron tener un padre que consume tabaco y la edad (Tabla 3).

Las variables que resultaron estadísticamente significativas en relación al consumo actual de tabaco fueron: el nivel de estudios, escala militar, padre consume tabaco y edad. Las variables nivel de estudio y tener un padre que consume tabaco conforman el modelo predictivo para el consumo actual de tabaco (Tabla 3).

Las variables que resultaron estadísticamente significativas en relación al consumo de cannabis fueron: localidad

Tabla 1. Características de la muestra y consumo de drogas en familiares y amigos

\begin{tabular}{lr}
\hline Sexo & $\%(\mathrm{n})$ \\
\hline Hombre & $93,9(568)$ \\
Mujer & $6,1(37)$ \\
Nivel de estudios & $\%(\mathrm{n})$ \\
\hline Estudios primarios & $21(127)$ \\
Estudios secundarios & $69,8(420)$ \\
Estudios universitarios & $9,1(55)$ \\
Con quién viven & $\%(\mathrm{n})$ \\
\hline Familia de origen & $40,2(243)$ \\
Vivienda compartida & $24,5(148)$ \\
Familia actual & $25(151)$ \\
Solo & $10,3(62)$ \\
Localidad de origen & $\%(\mathrm{n})$ \\
\hline Rural & $75(454)$ \\
Urbano & $25(151)$ \\
Unidad militar & $\%(\mathrm{n})$ \\
\hline Fuerza & $37,7(331)$ \\
\hline Cuartel general & $8,3(50)$ \\
\hline
\end{tabular}

\begin{tabular}{lr}
\hline Escala militar & $\%(\mathrm{n})$ \\
\hline Tropa & $83,5(505)$ \\
Mandos & $16,5(100)$ \\
Padre consume tabaco & $\%(\mathrm{n})$ \\
\hline
\end{tabular}

Si consume

41,7 (251)

Madre consume tabaco

$\%(n)$

Si consume

21,9 (132)

Hermano consume tabaco

$\%(n)$

Si consume

54,8 (328)

Amigos consumen drogas ilegales

$\%(n)$ 
Tabla 2. Prevalencias del consumo de drogas legales e ilegales

\begin{tabular}{lccccc}
\hline & & & \multicolumn{2}{c}{ Intervalo de confianza al 95\% } \\
\cline { 4 - 6 } & & Prevalencia (\%) & $\mathrm{n}$ & Límite inferior & Límite superior \\
\hline Consume de alcohol habitualmente & Si consumo & 39,9 & 241 & 36,1 & 43,9 \\
Consume de tabaco actualmente & Si consumo & 54,2 & 328 & 50,2 & 58,1 \\
Consumo de cannabis & Si consumo & 36,2 & 219 & 32,5 & 40,1 \\
Consumo de anfetaminas & Si consumo & 12,1 & 73 & 9,7 & 14,9 \\
Consumo de cocaína & Si consumo & 14,9 & 90 & 12,3 & 17,9 \\
Consumo de otras sustancias & Si consumo & 8 & 48 & 6 & 10,4 \\
\hline
\end{tabular}

Tabla 3. Análisis de regresión logística binaria para variables que predicen el consumo de drogas legales e ilegales

\begin{tabular}{|c|c|c|c|}
\hline & \multirow[b]{2}{*}{ Odds Ratio } & \multicolumn{2}{|c|}{ Intervalo de confianza al 95\% } \\
\hline & & Límite inferior & Límite superior \\
\hline \multicolumn{4}{|l|}{ Consumo habitual de alcohol } \\
\hline Padre consume tabaco (cr. no consume) & 1,5 & 1,0 & 2,1 \\
\hline Amigos consumen drogas ilegales (cr. no consume) & 1,4 & 1,0 & 2,0 \\
\hline Edad $^{a}$ & 0,96 & 0,93 & 0,99 \\
\hline \multicolumn{4}{|l|}{ Consumo actual de tabaco } \\
\hline Estudios de secundaria (cr. primarios) & 0,9 & 0,6 & 1,3 \\
\hline Estudios universitarios (cr. primarios) & 0,4 & 0,2 & 0,8 \\
\hline Padre consume tabaco (cr. no consume) & 1,4 & 1,0 & 2,0 \\
\hline \multicolumn{4}{|l|}{ Consumo de cannabis } \\
\hline Urbano (cr. rural) & 0,6 & 0,4 & 0,9 \\
\hline Apoyo a la fuerza (cr. fuerza) & 0,6 & 0,4 & 0,9 \\
\hline Cuartel general (cr. fuerza) & 1,2 & 0,6 & 2,3 \\
\hline Consumo de tabaco padre (cr. no consume) & 1,6 & 1,1 & 2,2 \\
\hline Amigos consumen drogas ilegales (cr. no consume) & 2,3 & 1,6 & 3,4 \\
\hline Edad & 0,9 & 0,9 & 1,0 \\
\hline Apoyo social & 0,6 & 0,4 & 0,9 \\
\hline \multicolumn{4}{|l|}{ Consumo de anfetaminas } \\
\hline Amigos consumen drogas ilegales (cr. no consume) & 5,5 & 2,8 & 10,7 \\
\hline Edad & 0,8 & 0,7 & 0,9 \\
\hline Apoyo social & 0,4 & 0,2 & 0,6 \\
\hline \multicolumn{4}{|l|}{ Consumo de cocaína } \\
\hline Estudios de secundaria (cr. primarios) & 0,6 & 0,3 & 1,0 \\
\hline Estudios universitarios (cr. primarios) & 0,2 & 0,0 & 1,3 \\
\hline Apoyo a la fuerza (cr. fuerza) & 0,4 & 0,2 & 0,7 \\
\hline Cuartel general (cr. fuerza) & 0,1 & 0,0 & 0,9 \\
\hline Madre consume tabaco (cr. no consume) & 1,7 & 1,0 & 2,9 \\
\hline Amigos consumen drogas ilegales (cr. no consume) & 2,8 & 4,9 & 8,7 \\
\hline Apoyo social & 0,4 & 0,2 & 0,7 \\
\hline \multicolumn{4}{|l|}{ Consumo de otras sustancias } \\
\hline Amigos consumen drogas ilegales (cr. no consume) & 8,7 & 3,8 & 19,9 \\
\hline Apoyo social & 0,4 & 0,2 & 0,7 \\
\hline
\end{tabular}

Nota. $c$ r. = categoría de referencia para la variable predictora; $\mathrm{a}=$ se presentan dos decimales pues si se redondea a un decimal se podría realizar una interpretación incorrecta. 
de origen, el nivel de estudios, con quién viven, unidad militar, escala militar, madre consume tabaco, padre consume tabaco, amigos consumen drogas ilegales, edad, habilidad de afrontamiento y apoyo social. Las variables que predicen significativamente el consumo de cannabis fueron: localidad de origen, unidad militar, padre consume tabaco, amigos consumen drogas ilegales, edad y apoyo social (Tabla 3).

Las variables que resultaron estadísticamente significativas en relación al consumo de anfetaminas fueron: el nivel de estudios, con quién viven, unidad militar, madre consume tabaco, amigos consumen drogas ilegales, edad y apoyo social. En el consumo de anfetaminas, las variables predictoras fueron amigos consumen drogas ilegales, edad y apoyo social (Tabla 3 ).

Las variables que resultaron estadísticamente significativas en relación al consumo de cocaína fueron: el nivel de estudios, con quién viven, unidad militar, escala militar, madre consume tabaco, padre consume tabaco, amigos consumen drogas ilegales, edad y apoyo social. Los resultados de las variables predictoras significativas que conforman el modelo para el consumo de cocaína fueron: unidad militar, escala militar, amigos consumen drogas y apoyo social (Tabla 3).

Las variables que resultaron estadísticamente significativas en relación al consumo de otras sustancias eran: escala militar, madre consume tabaco, amigos consumen drogas ilegales, edad, habilidad de afrontamiento y apoyo social. Las variables que predicen el consumo de otras sustancias eran amigos consumen drogas ilegales y apoyo social (Tabla 3).

\section{Discusión}

En el presente estudio, el tabaco era la droga más usada por la población militar española destinada en la zona de operaciones multinacionales de Bosnia- Herzegovina. El alcohol fue la segunda droga de mayor prevalencia de uso, seguida por el cannabis. La variable apoyo social era un importante predictor del consumo de drogas ilegales, de forma que un mayor apoyo social estaba asociado con una menor probabilidad de consumo. Otra variable también relevante que predecía el consumo de drogas fue tener amigos consumidores de drogas ilegales, es decir, en los militares con amigos consumidores de drogas ilegales se incrementaba la probabilidad de consumir ese tipo de drogas.

Como se ha comentado previamente, las drogas más consumidas en la zona de operaciones multinacionales fueron el tabaco (54,2\% consumen tabaco actualmente) y el alcohol (39,9\% consumen habitualmente alcohol). Con respecto a esta última droga, es importante mencionar que según parece el consumo de alcohol se incrementa en población militar desplegada en zona de operaciones, lo cual coincide con las conclusiones a las que llegan otros estudios (Jacobson et al., 2008; Kelsall et al., 2015). Los resultados obtenidos de un mayor consumo de drogas lega- les frente a las ilegales coinciden con otras investigaciones realizadas con población militar española no desplegada (Donoso Rodríguez, 2012). Por otro lado, si comparamos estos datos con los observados en la Encuesta Domiciliaria sobre Alcohol y Drogas en España (EDADES), seleccionando para ello el grupo de población civil y de la evaluación de consumo más próximas a la usada en el actual estudio, el 7,2\% de la población masculina con una edad de 25 a 34 años consumen alcohol diariamente. Con respecto al consumo de tabaco, el 37,9\% de la población masculina general de 25 a 34 años han consumido esta droga a diario en los últimos 30 días (Observatorio Español de la Droga y las Toxicomanías, 2015). En consecuencia, tentativamente se podría concluir que el consumo de tabaco y alcohol es superior en la población militar desplegada en zona de operaciones en comparación con la población civil espanola. No obstante, esta afirmación tendría que ser corroborada en futuros estudios donde se estén usado criterios de consumo similares.

Con respecto a las drogas ilegales, la más consumida era el cannabis donde un $36,2 \%$ de los militares la han probado alguna vez en la vida. La segunda más usada fue la cocaína con un 14,9\%, seguida de las anfetaminas con un $12,1 \%$. Un $8 \%$ de los militares participantes han probado otras sustancias. En un estudio realizado por Martínez et al. (2010) con población militar española no desplegada, el orden de las drogas ilegales más consumidas que encontraron en su muestra, era similar al obtenido en el presente estudio (el 9\% consumían actualmente cannabis, el $8 \%$ cocaína y el 3,5\% anfetaminas). Las prevalencias de consumo de drogas ilegales en el estudio de Martínez et al. (2010) eran inferiores a los obtenidos en zona de operaciones, aunque estos valores no serían del todo comparables pues cada estudio ha usado una medida de consumo diferente, "consumo actual" en la investigación realizada por Martínez et al. (2010) y "haber probado alguna vez en la vida" en el presente estudio. Otro aspecto a resaltar del estudio de Martínez et al. (2010) es que constataron que la prevalencia del consumo de drogas en población militar española se ha ido reduciendo a lo largo de los años (2002-2007) a excepción del año en el que se obtuvieron los datos de esta investigación, año en el que se produjo un incremento en el consumo de drogas ilegales respecto al año anterior. En la encuesta EDADES, las prevalencias de consumo de cannabis y cocaína en alguna ocasión en la vida eran superiores en población civil española de similar edad y sexo (población masculina con una edad de 25 a 34 años) a los obtenidos en población militar desplegada, en cambio, el consumo de anfetaminas era inferior al encontrado en la presente muestra. Concretamente, se obtuvo un consumo de un $50,5 \%$ en cannabis, un $9,4 \%$ en anfetaminas y un $22,1 \%$ en cocaína en población civil española (datos proporcionados por la Delegación del Gobierno para el Plan Nacional sobre Drogas según el estudio publicado por el 
Observatorio Español de la Droga y las Toxicomanías en el año 2015).

Los participantes cuyos padres consumían tabaco tenían mayor probabilidad de consumir alcohol, por el contrario, en los militares de mayor edad la probabilidad de uso disminuía. Estos resultados coinciden con los obtenidos por otros autores (Engels, Knibbe, de Vries, Drop y van Breukelen, 1999), los cuales indican que el modelo paternal juega un papel relevante en un mayor consumo de drogas. Es cierto que en el estudio longitudinal de Engels et al. (1999) los participantes eran adolescentes (la muestra tenía una edad media de 12,4 años en la primera recogida de los datos y una media de 17,4 en la última), por lo que sería interesante corroborar en futuras investigaciones si el modelo paternal incrementa el consumo de alcohol con población militar. En relación a la variable edad, resultados similares se han obtenido en un estudio realizado por Iversen et al. (2007) en población militar, el consumo de alcohol era inferior en el grupo de mayor edad.

La variable que se asociaba al incremento de consumo de tabaco fue tener padres consumidores de tabaco y la que se relacionaba con una reducción de la probabilidad de uso, era tener estudios universitarios. Es decir, los participantes con estudios universitarios tenían menos probabilidad de consumir tabaco que aquellos con estudios primarios. Al igual que en el consumo de alcohol, en un estudio longitudinal realizado por Brook et al. (2009) con población no militar, encontraron que el consumo de tabaco del padre estaba asociado con un incremento del propio consumo en la misma sustancia. Otra variable importante, en este caso asociada a protección, es tener estudios superiores. Resultados similares fueron encontrados en un estudio con población civil donde los participantes con un mayor nivel educativo tenían menor probabilidad de consumo de tabaco (Schnohr et al., 2004). Por lo tanto, el nivel de estudios es un factor relevante a tener en consideración pues en el presente trabajo sólo el 9.1\% de los participantes tenían estudios superiores.

El consumo de cannabis era menos probable cuando los participantes vivían en ambientes urbanos, pertenecían al grupo de apoyo a la fuerza, tenían una mayor edad, un mayor apoyo social. Un incremento en el consumo se producía cuando los padres consumían tabaco o sus amigos usaban drogas ilegales. Estos resultados son consistentes con los hallados por Coomber et al. (2011), donde se encontró que vivir en ambientes urbanos reducía la probabilidad de consumo de cannabis. De las variables específicas del ámbito militar, los participantes que pertenecían al grupo de apoyo a la fuerza tenían una probabilidad menor de consumo frente aquellos que pertenecen al grupo de la fuerza. Además, nuevamente se observa que el consumo de tabaco de los padres o tener una mayor edad son variables asociadas con el consumo de drogas, en esta ocasión, la primera con un incremento y la segunda con un decremento del uso de cannabis. En el informe elaborado por el Observatorio Español de la Droga y las Toxicomanías (2015) se encontró en población civil española similar tendencia, el consumo de cannabis está menos extendido entre los más mayores. Otro factor que parece importante fue el consumo de drogas ilegales por parte de amigos, confirmándose la influencia del grupo de amigos en el consumo al igual que se ha obtenido en otros estudios con población no militar (Rudzinski et al., 2014). El apoyo social era otra variable relevante, ya que un mayor apoyo social estaba asociado a una disminución de la probabilidad de consumo de cannabis. Resultados similares se han encontrado con población civil donde el apoyo social se relacionaba en idéntica dirección con el uso de cannabis (Chauchard, Septfons, y Chabrol, 2013).

La probabilidad de consumo de anfetaminas fue mayor en aquellos participantes cuyos amigos consumían drogas ilegales, y menor en militares de mayor edad o con más apoyo social. Resultados similares se han encontrado en otros colectivos (Observatorio Español de la Droga y las Toxicomanías, 2015; Rice, Milburn, Rotheram-Borus, Mallett y Rosenthal, 2005).

Algunas variables específicas del ámbito militar estaban asociadas con el consumo de cocaína. Según parece, los participantes cuya función era apoyar a la fuerza tienen menos probabilidad de consumir que aquellos que pertenecen al grupo de la fuerza. Resultados similares se obtenían cuando se comparaba al grupo que pertenece al cuartel general con el grupo de la fuerza, observándose un menor consumo en los miembros del cuartel general. En el estudio realizado por Donoso Rodríguez (2012) encontraron también un menor consumo en el cuadro de mandos. Quizás, estos resultados se deban al hecho de que el personal que conforma la fuerza ha sido adiestrado para emitir un comportamiento algo más temerario, y, por consiguiente, para afrontar los retos que suponen las misiones críticas con una actitud más desinhibida que el personal del cuartel general y apoyo a la fuerza, el cual se dedica casi exclusivamente la toma de decisiones y a tareas logísticas. Comportamiento y actitudes desinhibidos que podrían generalizarse a otros aspectos de la vida, como lo puede ser en este caso la transgresión de la norma de no consumo de drogas ilegales. Una variable que se asociaba a un incremento del consumo de cocaína era tener amigos consumidores de drogas ilegales, y relacionada con una disminución de su uso era contar con un mayor apoyo social. En otro estudio con población no militar se ha obtenido también una asociación de estas variables con el consumo de cocaína (Bohnert, German, Knowltonc y Latkinc, 2010).

En relación con el consumo de otras sustancias, las variables predictoras fueron las dos que han sido relevantes en todas las drogas ilegales. Esto es, tener amigos que consumen drogas ilegales aumenta la probabilidad de uso de otras sustancias, y un mayor apoyo social disminuye el consumo. 
Entre las limitaciones de la presente investigación se indican las siguientes: a) haber evaluado a participantes de una única zona de operaciones multinacionales, ya que las exigencias de las diferentes zonas de despliegue podrían demandar diferentes recursos profesionales y psicológicos; b) tener un valor de fiabilidad en la escala de apoyo social muy próximo al mínimo recomendable; c) aplicar un diseño transversal que no permite valorar la evolución del consumo; d) utilizar sólo medidas subjetivas del consumo de drogas. Por tanto, en futuras investigaciones sería recomendable evaluar al personal militar destinado en distintas zonas de operaciones multinacionales y compararlas con población militar española no desplegada utilizando para ello medidas de consumo similares. También sería conveniente aplicar diseños longitudinales a efecto de poder evaluar el consumo de drogas en distintos momentos temporales y, además, acompañar las evaluaciones subjetivas con otras objetivas que puedan aportar información inequívoca de consumo utilizando, por ejemplo, analíticas de sangre y orina.

En conclusión, hay consumo de drogas en la zona de operaciones multinacionales, y este uso parece incrementarse cuando se tiene amigos consumidores de drogas ilegales y disminuye cuando se dispone de un mayor apoyo social. Por tanto, sería importante aplicar medidas de prevención teniendo en cuenta que dicho consumo podría afectar a un desempeño adecuado de las misiones encomendadas. Por ejemplo, podría incrementarse la oferta de actividades saludables de ocio y tiempo libre, sobre todo si se tiene en cuenta el número de horas libres disponibles fuera del horario dedicado a la actividad profesional.

\section{Agradecimientos}

Nuestro agradecimiento a la Delegación del Gobierno para el Plan Nacional sobre Drogas por habernos facilitado información de gran utilidad para la realización de este trabajo.

\section{Conflicto de intereses}

No existen conflictos de intereses.

\section{Referencias}

Bohnert, A. S. B., German, D., Knowltonc, A. R. y Latkinc, C. A. (2010). Friendship networks of inner-city adults: A latent class analysis and multi-level regression of supporter types and the association of supporter latent class membership with supporter and recipient drug use. Drug and Alcohol Dependence, 107, 134-140. doi:10.1016/j. drugalcdep.2009.09.012.

Brook, J. S., Saar, N. S., Zhang, C. y Brook, D. W. (2009). Familial and non-familial smoking: Effects on smoking and nicotine dependence. Drug and Alcohol Dependence, 101, 62-68. doi:10.1016/j.drugalcdep.2008.11.003.

Chauchard, E., Septfons, A. y Chabrol, H. (2013). Motivations et stratégies lors d'arrêt spontané de la consommation de cannabis: quel impact sur les rechutes? L'Encéphale, 39, 385-392. doi:10.1016/j.encep.2013.03.008.

Coomber, K., Toumbourou, J. W., Miller, P., Staiger, P. K., Hemphill, S. A. y Catalano, R. F. (2011). Rural adolescent alcohol, tobacco, and illicit drug use: A comparison of students in Victoria, Australia, and Washington state, United States. The Journal of Rural Health, 27, 409-415. doi:10.1111/j.1748-0361.2010.00360.x.

Cucciare, M. A., Sadler, A. G., Mengeling, M. A., Torner, J. C., Curran, G. M., Han, X. y Booth, B. M. (2015). Associations between deployment, military rank, and binge drinking in active duty and reserve/national guard US servicewomen. Drug and Alcohol Dependence, 153, 37-42. doi:10.1016/j.drugalcdep.2015.06.013.

Donoso Rodríguez, D. (2012). Epidemiología en drogas. Análisis de tendencias de consumo de sustancias psicotrópicas en el ejército de tierra (1997-2007). En D. Donoso Rodríguez (Ed.), Psicología en las Fuerzas Armadas (pp. 207-228). Madrid, España: Ministerio de Defensa.

Engels, R. C. M. E., Knibbe, R. A., de Vries, H., Drop, M. J. y van Breukelen, G. J. P. (1999). Influences of parental and best friends' smoking and drinking on adolescent use: A longitudinal study. Journal of Applied Social Psychology, 29, 337-361. doi:10.1111/j.1559-1816.1999. tb01390.x.

Iversen, A., Waterdrinker, A., Fear, N., Greenberg, N., Barker, C., Hotopf, M., ... Wessely, S. (2007). Factors associated with heavy alcohol consumption in the U.K. armed forces: Data from a health survey of Gulf, Bosnia, and era veterans. Military Medicine, 172, 956-961. doi:10.7205/MILMED.172.9.956.

Jacobson, I. G., Ryan, M. A. K., Hooper, T. I., Smith, T. C., Amoroso, P. J., Boyko, E. J., ... Bell, N. S. (2008). Alcohol use and alcohol-related problems before and after military combat deployment. JAMA, 300, 663-675. doi:10.1001/jama.300.6.663.

Kelsall, H. L., Wijesinghe, M. S. L., Creamer, M. C., McKenzie, D. P., Forbes, A. B., Page, M. J. y Sim, M. R. (2015). Alcohol use and substance use disorders in Gulf War, Afghanistan, and Iraq War veterans compared with nondeployed military personnel. Epidemiologic Reviews, 37, 38-54. doi:10.1093/epirev/mxu014.

Martínez, M., Alonso, V., Taranco, M. y Gutiérrez, C. (2010). Encuesta sobre drogas a los militares de tropa y marinería de las Fuerzas Armadas españolas. Sanidad Militar, 66, 70-82.

Martínez-González, J. M., Trujillo-Mendoza, H. M. y Robles-Lozano, L. (2007). Factores de riesgo, protección y representaciones sociales sobre el consumo de drogas: implicacio- 
nes para la prevención. Sevilla, España: Consejería para la Igualdad y el Bienestar Social de la Junta de Andalucía.

Melchior, M., Chastang, J.-F., Goldberg, P. y Fombonne, E. (2008). High prevalence rates of tobacco, alcohol and drug use in adolescents and young adults in France: Results from the GAZEL youth study. Addictive Behaviors, 33, 122-133. doi:10.1016/j.addbeh.2007.09.009-

Miquel, L., Rodamilans, M., Giménez, R., Cambras, T., Canudas, A. M. y Gual, A. (2015). Evaluación del consumo de riesgo de alcohol en estudiantes universitarios de la Facultad de Farmacia. Adicciones, 27, 190-197. doi:10.20882/adicciones.705.

Motos Sellés, P., Cortés Tomás, M.T., Giménez Costa, J. A. y Cadaveira Mahía, F. (2015). Predictores del consumo semanal de alcohol y sus consecuencias asociadas en universitarios consumidores intensivos de alcohol. Adicciones, 27, 119-131. doi:10.20882/adicciones.700.

Mounteney, J., Griffiths, P., Sedefov, R., Noor, A., Vicente, J. y Simon, R. (2016). The drug situation in Europe: an overview of data available on illicit drugs and new psychoactive substances from European monitoring in 2015. Addiction, 111, 34-48. doi:10.1111/add.13056.

Newcombe, R. G. (1998). Two-Sided Confidence Intervals for the Single Proportion: Comparison of Seven Methods. Statistics in Medicine, 17, 857-872. doi:10.1002/ (SICI) 1097-0258(19980430) 17:83.0.CO;2-E.

Observatorio Español sobre Drogas (2013). Encuesta escolar sobre uso de drogas en estudiantes de Enseñanzas Secundarias (ESTUDES) 2012-2013. Madrid, España: Ministerio de Sanidad, Servicios Sociales e Igualdad.

Observatorio Español de la Droga y las Toxicomanías (2015). Informe 2015. Alcohol, tabaco y drogas ilegales en España. Madrid, España: Ministerio de Sanidad, Servicios Sociales e Igualdad.

Observatorio Europeo de las Drogas y las Toxicomanías (2014). Informe europeo sobre drogas. Tendencias y novedades. Luxemburgo, Luxemburgo: Observatorio Europeo de las Drogas y las Toxicomanías.

Rice, E., Milburn, N. G., Rotheram-Borus, M. J., Mallett, S. y Rosenthal, D. (2005). The effects of peer group network properties on drug use among homeless youth. The American Behavioral Scientist, 48, 1102-1123. doi:10.1177/0002764204274194.

Rudzinski, K., Dawe, M., McGuire, F., Shuper, P. A., Rehm, J. y Fischer, B. (2014). Reflections regarding future cannabis use among high-frequency users in a Canadian university student population. Journal of Research on Adolescence, 24, 598-607. doi:10.1111/jora.12087.

Schnohr, C., Højbjerre, L., Riegels, M., Ledet, L., Larsen, T., Schultz-Larsen, K., ... Grønbaek, M. (2004). Does educational level influence the effects of smoking, alcohol, physical activity, and obesity on mortality? A prospective population study. Scandinavian Journal of Public Health, 32, 250-256. doi:10.1080/14034940310019489.
Sordo, L., Indave, B. I., Pulido, J., Molist, G., Rosales-Statkus, M. E., Ruiz-García, M. y Barrio, G. (2015). Epidemiología del abuso de alcohol entre la población inmigrante en España. Adicciones, 27, 132-140. doi:10.20882/ adicciones.697.

Teachman, J., Aderson, C. y Tedrow, L. M. (2015). Military service and alcohol use in the United States. Armed Forces E Society, 41, 460-476. doi:0.1177/0095327X14543848.

Unidad de Estadística del Órgano Central (2016). Estadística del personal militar de complemento, militar de tropa y marinería y reservista voluntario 2015. Madrid, España: Ministerio de Defensa.

Wilkinson, L. y APA Task Force on Statistical Inference. (1999). Statistical methods in psychology journal: Guidelines and explanations. American Psychologist, 54, 594604. doi:10.1037/0003066X.54.8.594. 